\title{
Integrated Extraction and Catalytic Upgrading of Bio-Crude Oil from Co-Hydrothermal Liquefaction of Crude Glycerol and Algae
}

Zheng Cui ${ }^{1}$, Feng Cheng ${ }^{2}$, Jacqueline M. Jarvis ${ }^{3}$, Umakanta Jena ${ }^{1 *}$, Catherine E. Brewer ${ }^{1}$

Supplemental information

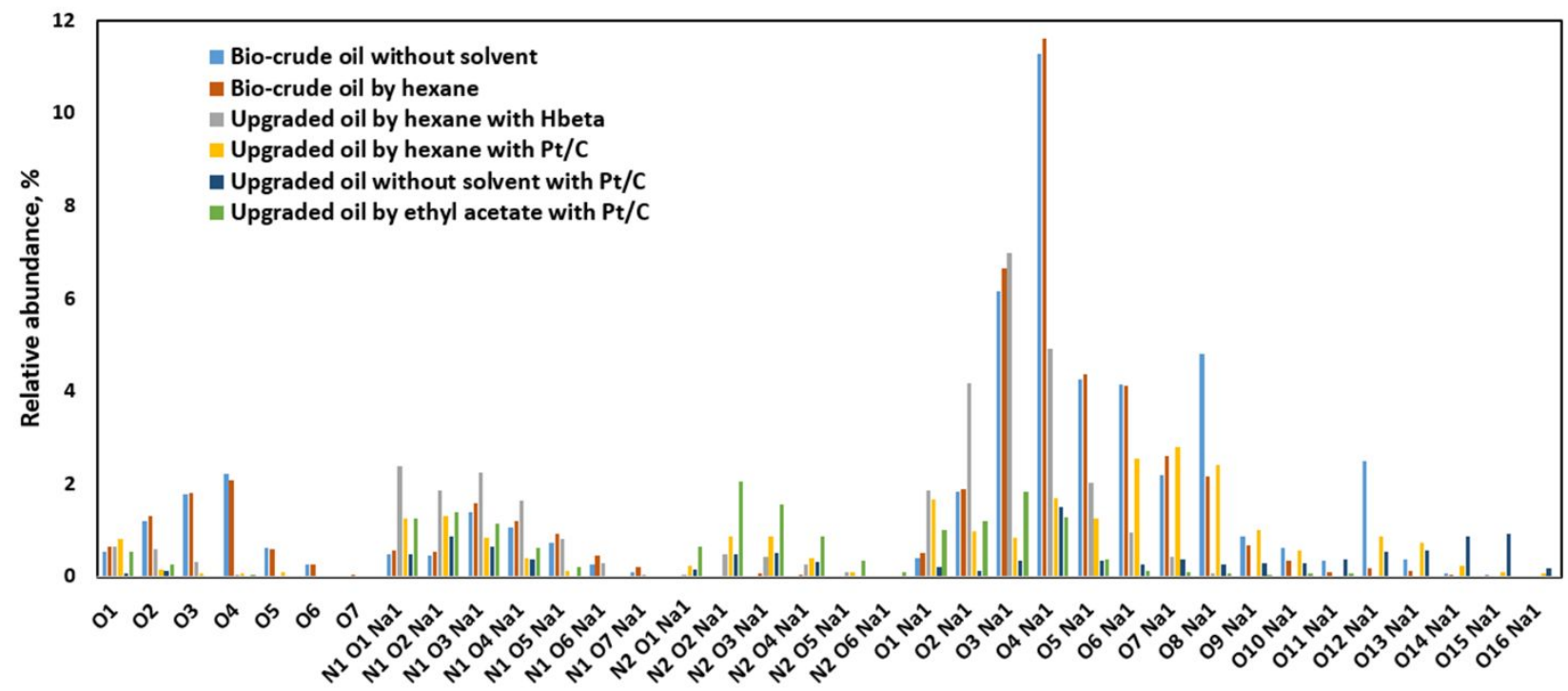

(a)

Heteroatom class

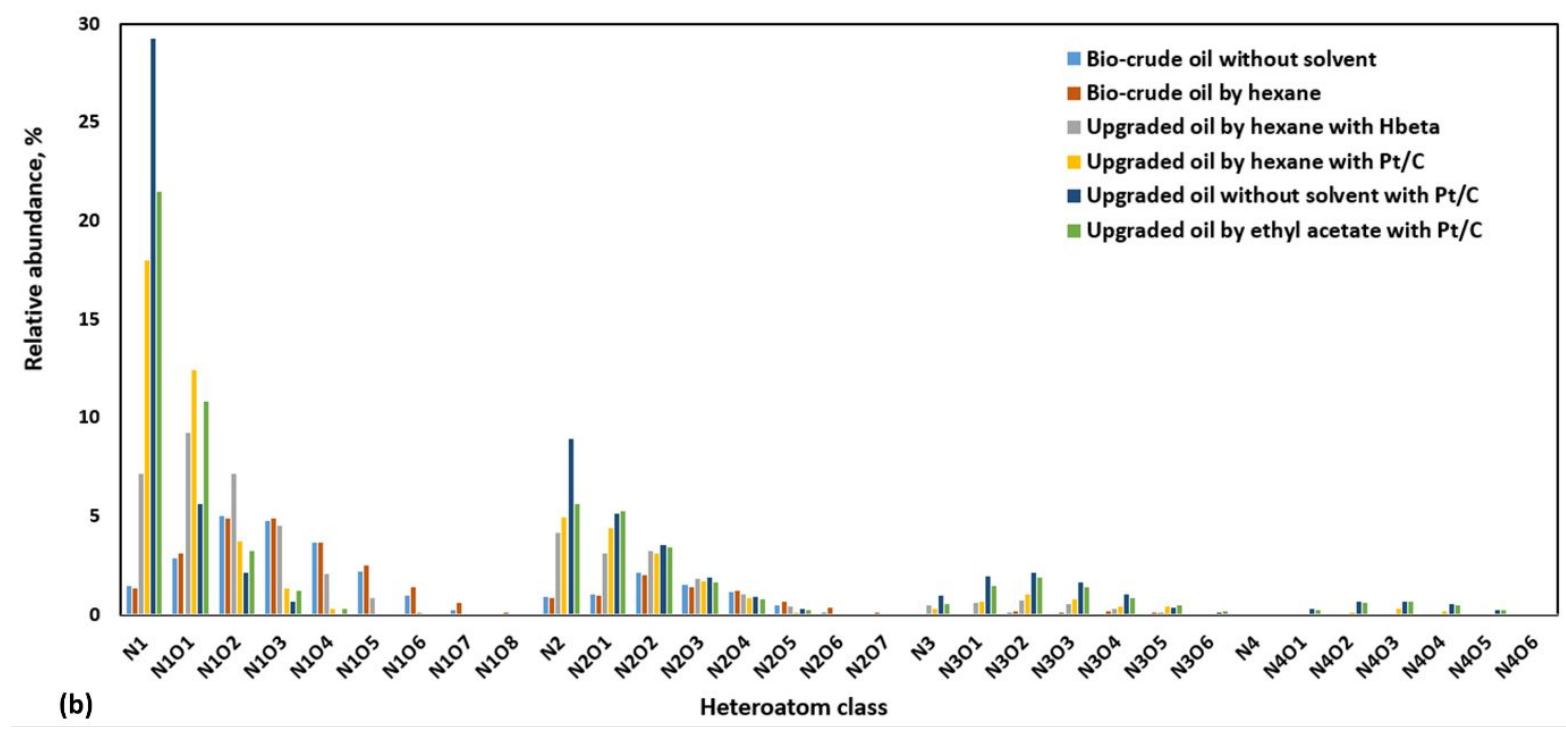


Figure S1. Detailed heteroatom class distributions of extracted bio-crude oils and upgraded oils from extraction and/or upgrading with different solvents and catalysts; data derived from positive-ion ESI FT-ICR MS. 